\title{
Place des micro-organismes atypiques et des virus dans les pneumonies aiguës communautaires sévères
}

\section{The role of atypical microorganisms and viruses in severe acute community-acquired pneumonia}

\author{
B. Planquette $\cdot$ A. Ferré $\cdot$ J.-P. Bédos
}

Reçu le 22 octobre 2012 ; accepté le 21 novembre 2012

(C) SRLF et Springer-Verlag France 2012

Résumé L'attention des réanimateurs n'est guère attirée par les bactéries atypiques ou les virus dans le cadre des pneumonies communautaires sévères. Seules classiquement, Legionella pneumophila et les virus influenza, du fait de la pandémie grippale récente $\mathrm{H} 1 \mathrm{~N} 1$, restent présents dans la réflexion diagnostique. Pourtant, les pneumonies bactériennes atypiques ne sont pas rares, représentant jusqu'à $44 \%$ des pneumonies communautaires. Considérées comme moins sévères que les pneumonies à bactéries pyogènes « typiques », $25 \%$ d'entre elles justifient une hospitalisation, et $15 \%$ présentent des critères de sepsis sévères. Si Legionella pneumophila est plus fréquemment en cause dans les formes graves, Mycoplasma pneumoniae peut être responsable de véritables défaillances multiviscérales. Les outils diagnostiques sont aujourd'hui performants (antigénurie légionelle et recherche en polymerase chain reaction [PCR] de mycoplasme en particulier) et permettent un diagnostic rapide et précoce. Le traitement est bien codifié et repose sur les macrolides ou les fluoroquinolones, en association dans les formes graves de légionellose. Le rôle des virus au cours des pneumonies communautaires sévères, seuls ou associés à des bactéries, devient de plus en plus établi grâce aux méthodes de diagnostic rapide de biologie moléculaire. Ces techniques ont permis la mise en évidence dans ces pneumonies de nombreux et nouveaux virus. Toute la question est désormais à l'imputabilité clinique de ces résultats biologiques très performants et donc à la décision d'instaurer ou non un traitement antiviral dont l'efficacité dans les formes sévères reste discutable.

Mots clés Pneumonie aiguë communautaire grave Détresse respiratoire $\cdot$ Bactérie atypique $\cdot$ Légionellose Pneumonie virale

\footnotetext{
B. Planquette $(\triangle) \cdot$ A. Ferré $\cdot$ J.-P. Bédos Service de réanimation médicochirurgicale, centre hospitalier de Versailles, site Mignot, 177, rue de Versailles, F-78150 Le Chesnay, France e-mail : benjamin.planquette@gmail.com
}

\begin{abstract}
Usually, intensivists do not focus on atypical bacteria and viruses in severe community-acquired pneumonia (CAP). Only Legionella pneumophila and influenza virus, following the recent H1N1 influenza pandemic, are routinely suggested as responsible agents. However, CAP due to atypical bacteria may represent up to $44 \%$ of all CAP. Viral CAP is considered less severe than the usual bacterial ones, although $25 \%$ of them warrant hospitalization and $15 \%$ result in severe sepsis. Even though L. pneumophila is the most frequently atypical pathogen involved in severe cases, Mycoplasma pneumoniae may be responsible for multiorgan failure. To date, tools including detection of Legionella antigen in urine and Mycoplasma using polymerase chain reaction (PCR) allow rapid and accurate diagnosis. The treatment is based on macrolides and fluoroquinolones that can be associated in severe Legionnaire diseases. The presence of virus in CAP, either alone or in association with bacteria, has been demonstrated using molecular biology tests. These techniques also allowed the identification of several new viruses in CAP. However, the exact role of these detected viruses in CAP as well as the efficiency of antiviral therapy still represent major unsolved concerns.
\end{abstract}

Keywords Severe community-aquired pneumonia · Respiratory failure $\cdot$ Atypical pneumonia .

Legionella pneumophila $\cdot$ Viral pneumonia

\section{Introduction}

Les pneumonies aiguës communautaires (PAC) représentent $7 \%$ de la mortalité totale dans le monde [1]. Dans les PAC sévères de l'adulte, la fréquence des micro-organismes atypiques et des virus a toujours été considérée comme faible, Strepococcus pneumoniae restant le premier pathogène responsable de 20 à $30 \%$ de mortalité en réanimation [2]. Parmi les micro-organismes atypiques, Legionella pneumophila est le principal en termes de morbimortalité [3]. Ainsi, 
dans la prise en charge thérapeutique probabiliste d'une PAC sévère, il est recommandé d'associer des antibiotiques actifs sur ces deux micro-organismes [4]. Près de 100 millions de cas de PAC virales surviennent chaque année chez l'adulte dans le monde [5]. L'émergence en 2003 du syndrome aigu respiratoire sévère au Coronavirus (SARS) [6], de la grippe aviaire (virus influenza H5N1) [7] et, en 2009, de la pandémie grippale (virus influenza H1N1) [8] ont remis au premier plan le rôle possible des virus dans les PAC sévères de l'adulte immunocompétent. De plus, la mise en évidence de nouveaux virus respiratoires a relancé la question de leur imputabilité clinique [9]. Les fréquentes coinfections virus-bactéries posent le problème du bénéfice des antibiotiques et des antiviraux systématiques au cours de PAC virales [5].

\section{Pneumonies virales}

Responsables de PAC chez l'enfant, les virus à tropisme respiratoire sont de plus en plus fréquemment mis en évidence chez l'adulte, seuls ou associés à une infection bactérienne. Jusqu'à ces dernières années, la recherche d'une étiologie virale d'une PAC était négligée du fait de l'absence de thérapeutique antivirale ayant démontré une efficacité [10]. Les pneumonies virales sont plus fréquentes chez l'enfant, car celui-ci est le « réservoir naturel» de ces virus ; la contamination des adultes se fait par leur intermédiaire en milieu communautaire et par voie bronchogène après inhalation de particules oropharyngées infectées. Il n'existe aucun tableau clinique spécifique de pneumonie virale [5]. Il est classique de dire que le début est lent dans un contexte épidémique. Biologiquement, une leuconeutropénie est classique et la procalcitonine (PCT) est basse [11]. L'aspect radiologique est polymorphe et non spécifique.

\section{Méthodes diagnostiques}

L'un des progrès les plus importants dans le cadre de ces pneumonies concerne les méthodes de diagnostic virologique $[5,12]$. Les anciennes méthodes sont actuellement supplantées par les techniques de biologie moléculaire (amplification en Reverse transcriptase - polymerase chain reaction [RT-PCR] Multiplex) très sensibles. Elles détectent les ARN messagers de nombreux virus, témoins de leur réplication [13,14]. Grâce à ces techniques, au moins 26 virus ont été « associés » à des PAC. La réalité du diagnostic de pneumonie virale reste malgré tout un « challenge », car différencier un portage ou une colonisation d'une réelle infection et l'obtention d'un prélèvement restent difficiles. Plusieurs sites de prélèvement ont été étudiés [15], il semble que le lavage bronchoalvéolaire (LBA) soit la méthode de référence au cours des PAC sévères [16].
L'hétérogénéité des sites de prélèvement dans les publications explique la fluctuation de la prévalence des pneumonies virales (15-56\%) dans les études [5]. Néanmoins, un tiers des PAC de l'adulte serait associé à une infection virale. Seule une étude récente réalisée au cours de PAC sévères hospitalisées en réanimation a détecté de façon systématique la présence de virus par PCR, principalement dans le LBA [17].

\section{Aspect épidémiologique}

Trois études récentes, avec des collectifs de 304, 193 et 183 patients, ont analysé l'incidence d'une étiologie virale potentielle de PAC de l'adulte hospitalisées. La première étude, néo-zélandaise [18], retrouvait au moins un virus chez $29 \%$ de patients (âge médian de 70 ans). Les rhinovirus, virus influenza, le virus respiratoire syncytial (VRS) et les adénovirus étaient les plus fréquents. Il existait $16 \%$ de coinfections dont plus de $90 \%$ étaient une association virus-bactérie (pneumocoque, en premier lieu). Seulement $4 \%$ des patients nécessitaient une prise en charge en réanimation. La deuxième, réalisée aux États-Unis [19], retrouvait au moins un virus chez $15 \%$ des patients. Influenza A et B, Human metapneumovirus et VRS étaient les plus fréquents avec un taux de coinfections virus-bactérie de $4 \%$. Les patients avec infection virale étaient plus âgés, avec des comorbidités cardiaques, mais aucun n'a été pris en charge en réanimation. La troisième étude, réalisée en Israël [20], comparait en combinant plusieurs techniques de prélèvement 183 PAC virales à 450 patients « témoins » ambulatoires et 201 patients hospitalisés pour une bronchite. Au moins un virus était retrouvé chez $32 \%$ des patients ayant une PAC, chez $52 \%$ des patients ayant une bronchite et chez $7 \%$ des patients « témoins ». Dans les PAC, les coronavirus, VRS, rhinovirus et influenza A étaient les plus fréquents. Une association virale était retrouvée dans $18 \%$ des cas. $\mathrm{Au}$ regard de ces trois études, l'incidence des PAC virales hospitalisées semble croissante mais elles restent moins sévères et nécessitent rarement une admission en réanimation. Outre le virus influenza, d'autres virus " émergents » sont en cause. Qu'en est-il en réanimation ? La seule étude récente s'intéresse à une population de patients sévères hospitalisés en réanimation pour une PAC ou une pneumonie associée aux soins (HCAP) [17] avec un âge moyen de 65 ans, un diabète (27\%) et/ou une atteinte pulmonaire chronique $(31 \%), 92 \%$ de patients ventilés et $51 \%$ en état de choc. Une recherche microbiologique complète a été faite chez 198 patients (64 PAC et 134 HCAP). Un pathogène a été identifié chez $67 \%$ des patients (bactérie : $36 \%$, virus : $36 \%$, et association : $9 \%$ ) sans différence de fréquence entre PAC et HCAP. Un seul virus était retrouvé chez $87 \%$ des patients et deux virus chez $13 \%$ des patients. La fréquence des virus isolés était : rhinovirus : $24 \%$, 
Parainfluenza virus : $21 \%$, Human metapneumovirus : $18 \%$, influenza virus : $17 \%$, VRS : $14 \%$, cytomégalovirus (CMV) : $11 \%$, coronavirus OC43 : 5,6\%, et adénovirus/ entérovirus : $1,4 \%$. Seul le VRS était plus fréquent au cours des PAC (11\%) qu'au cours des HCAP $(2 \%)[p=0,01]$. De façon intéressante, $60 \%$ des patients étaient traités par un antiviral dans un délai médian de deux jours. Parmi les pneumonies virales identifiées, $25 \%$ avait une coinfection bactérienne principalement à pneumocoque (36\% des patients). La mortalité à 28 jours était de $35 \%$ pour les PAC. Les mortalités des PAC bactériennes, virales ou des HCAP étaient similaires respectivement de 25,26 et $33 \%$. Au cours de la pandémie grippale H1N1 en 2009 [8], une infection bactérienne secondaire était retrouvée dans 4 à $24 \%$ des cas. Malgré la réalisation de LBA chez $56 \%$ des patients dans cette étude, l'isolement de virus reposait sur des sécrétions nasopharyngées dans $44 \%$ des cas, posant le problème de la coïncidence possible entre une infection virale des voies aériennes supérieures et une PAC d'autre origine.

\section{Aspect thérapeutique}

Sur un plan thérapeutique, deux questions « théoriques» se posent [5] : faut-il utiliser des antiviraux et/ou des antibiotiques au cours des PAC virales sévères ? En pratique, toute PAC sévère doit recevoir, en traitement probabiliste urgent, une association d'antibiotiques intraveineux (IV) visant les bactéries typiques (pneumocoque en particulier) et les « atypiques » (L. pneumophila en particulier). La seconde question est : doit-on arrêter l'antibiothérapie et doit-on débuter un antiviral seul en cas de RT-PCR virale positive ? Aucun consensus n'existe vis-à-vis de la première question. Si un prélèvement de qualité documente une bactérie associée, une antibiothérapie adaptée est incontournable. Si, en revanche, il n'y a pas de documentation bactérienne, l'arrêt de l'antibiothérapie initiale reste très discuté et discutable, du fait de la fréquence des coinfections et de l'activité « aléatoire » des antiviraux. Si le patient est vierge d'antibiothérapie, que les prélèvements fiables initiaux ne retrouvent qu'une étiologie virale, ne pas mettre d'antibiotique reste un choix possible au sein d'une équipe et ne peut se concevoir qu'avec un suivi de grande qualité. Concernant le traitement antiviral, dans les PAC virales sévères, les données sont très limitées [10]. Il faut distinguer le virus influenza des autres virus pour lesquels nous n'avons que des expériences de cas isolés et quelques études chez des patients immunodéprimés. La ribavirine a un spectre large actif sur le VRS, l'Human metapneumovirus et les Pararinfluenza virus [21]. Le pléconaril a été utilisé en compassionnel au cours de PAC à rhinovirus et entérovirus. Une plus grande expérience existe avec les traitements pour les virus influenza, en particulier durant la pandémie H1N1 en 2009. Il existe deux inhibiteurs de neuraminidases, l'olsétamivir (oral) et le zanamivir (inhalation ou IV), qui étaient proposés en traitement précoce (dans les 48 heures du début des symptômes) des infections à virus influenza A ou B. Au cours de la pandémie H1N1, le bénéfice de l'olsétamivir a été clairement démontré [22] : maximale si le traitement est précoce, il persiste s'il est débuté au-delà des 48 premières heures [22].

\section{Formes cliniques selon le virus}

\section{Virus influenza (ou virus de la grippe, orthomyxovirus)}

Ce virus est classé en trois catégories $\mathrm{A}, \mathrm{B}$ ou $\mathrm{C}$; son virion a une enveloppe. Les variations de ces protéines définissent le type viral incriminé : H1N1, H2N2, H5N1... Le virus A est quasiment seul responsable des formes sévères lors d'épidémies ou de pandémies. Contrairement aux grandes pandémies de 1918, 1957 et 1968, les plus récentes n'ont pas eu une telle ampleur en termes de mortalité. La grippe aviaire H5N1 n'a causé qu'environ 450 infections en 2003, mais avec un taux élevé de mortalité de l'ordre de $60 \%$ [23]. Plus récemment, la pandémie grippale de 2009 à $\mathrm{H} 1 \mathrm{~N} 1$ a fait environ 18000 morts [24] avec des formes sévères différentes des précédentes : patients jeunes (âge médian de 40 ans) sans comorbidités, femmes enceintes, patients obèses. La mortalité en réanimation était de 15 à $40 \%$, malgré une prise en charge thérapeutique maximaliste (antiviraux spécifiques, techniques d'oxygénation extracorporelle [ECMO]) [25]. La corticothérapie dans ces syndromes de détresse respiratoire aiguë (SDRA) grippaux n'a pas fait la preuve de son efficacité ayant même aggravé la mortalité si elle était administrée précocement [26].

\section{VRS (famille des paramyxovirus)}

Il est la troisième cause de PAC virale hospitalisée, et survient par poussée épidémique de l'automne au printemps [27,28]. L'évolution vers le décès par SDRA est décrite surtout chez les personnes âgées ou lors d'épidémies en institution. Un traitement par ribavirine intraveineuse se discute.

\section{Adénovirus}

Une revue de la littérature récente retrouve 21 cas de PAC virale à adénovirus chez l'immunocompétent entre 1975 et 2008 [29] avec 57 \% d'hommes (âge médian de 40 ans) préférentiellement caucasiens et obèses. Les opacités interstitielles diffuses étaient plus fréquentes que les condensations alvéolaires. Une ventilation mécanique a été nécessaire chez $67 \%$ des patients avec une mortalité de $24 \%$. Des épidémies sont décrites dans des populations vivant en milieu fermé (casernes) [30,31]. 


\section{Coronavirus}

Les coronavirus représentent $5 \%$ des étiologies virales des PAC et ont été à l'origine de l'épidémie asiatique de SARS en 2003 [6,32]. Environ 20 à $30 \%$ des patients étaient admis en réanimation avec une mortalité globale de $10 \%$, mais de $50 \%$ chez les patients de plus de 65 ans.

\section{Parainfluenzae virus}

Les PAC à Parainfluenza virus sont occasionnelles chez l'adulte et rarement sévères [33].

\section{Entérovirus (famille des Picornaviridae)}

Ils sont ubiquitaires à transmission orofécale et survivent longtemps dans le milieu extérieur expliquant la prépondérance chez les enfants et dans les pays en voie de développement. Leur rôle pathogène n'est pas clair (10,5 \% de portage asymptomatique chez l'adulte [34]), mais la pneumonie à entérovirus est décrite [5] principalement chez les enfants [35]. Il existe des données contradictoires chez l'adulte immunocompétent. Une étude portant sur 231 patients avec une PAC retrouvait 5,2\% d'entérovirus avec $58 \%$ de coinfections (25\% de pneumocoque) [36]. Dans une étude récente regroupant 64 cas de PAC en réanimation, aucun entérovirus n'était retrouvé [17]. Cependant, une revue de morbimortalité a décrit des cas de PAC à Human Enterovirus 68 (HEV68) chez l'adulte, avec des SDRA possibles [37].

\section{Human bocavirus (HBoV, famille des Parviviridae)}

Le HBoV, identifié en 2005, est ubiquitaire. Quatre types ont été identifiés ( 1 à 4 ), et le type 1 semble prépondérant chez l'homme sans que le mode de transmission ne soit clair. La présentation clinique est aspécifique [38], et le diagnostic se fait par RT-PCR. Certaines séries rapportent des infections des voies aériennes à $\mathrm{HBoV}$, mais son rôle pathogène n'est pas élucidé : $94 \%$ des adultes ont été en contact avec ce virus [39], il est retrouvé chez $43 \%$ d'enfants asymptomatiques [40]. Il est souvent associé à d'autres pathogènes viraux lors d'infections des voies aériennes [41]. HBoV reste une cause rare de pneumonie chez l'adulte [5] : de 0,8\% chez des patients ayant une PAC ou une exacerbation de bronchopneumopathie chronique obstructive (BPCO) [40] à $1 \%$ dans une série d'adultes hospitalisés pour une PAC [42]. Dans l'étude récente en réanimation, sur les 64 cas de PAC, aucun HBoV n'a été retrouvé [17]. À noter que la majorité des études n'incluait pas une recherche systématique du HBoV. La présentation clinicoradiologique est aspécifique sans critère de gravité dans la majorité des cas [43]. Le traitement reste symptomatique.

\section{Métapneumovirus humain (hMPV)}

C'est un virus ubiquitaire qui sévit sous forme épidémique (pics hivernaux). Certaines études retrouvent une séroprévalence de près de $99 \%$ chez les jeunes adultes [44]. Une réinfection est possible à l'âge adulte après exposition à un génotype différent ou du fait d'un déclin de l'immunité [45]. Chez les immunocompétents, hMPV semble toucher de manière plus agressive les sujets fragiles (jeunes enfants, personnes âgées) pouvant être responsables de bronchiolites ou de PAC graves. Il serait en cause dans 2 à $5 \%$ des affections pseudogrippales, des PAC, des exacerbations aiguës de pathologies respiratoires chroniques [46]. Dans une cohorte américaine de 1386 patients hospitalisés pour une infection de voies respiratoires ( $25 \%$ de PAC), 6,6\% étaient infectés par le hMPV (30\% de coinfections). La moitié de ces pneumonies à hMPV nécessitaient la mise sous assistance ventilatoire avec $25 \%$ de mortalité (85 ans de moyenne d'âge pour les patients décédés) [47]. Une autre étude regroupant 193 PAC retrouvait $4 \%$ de hMPV, mais cette fois sans critère de gravité clinique ni décès [48]. Lorsqu'une infection virale est documentée, la prévalence de hMPV est de $24 \%$ dans le cadre de PAC et $8 \%$ en cas de PAC grave $[17,48]$. Cliniquement aspécifique, le traitement est symptomatique. Certaines données in vitro et sur modèles animaux suggèrent une efficacité de la ribavirine ; mais ces données sont insuffisantes pour recommander ce traitement en pratique clinique [49].

\section{Pneumonie varicelleuse (varicella-zona virus ou VZV)}

La varicelle est fréquente chez les enfants dans les pays où la vaccination n'est pas la règle; mais elle est plus grave chez l'adulte $(<5 \%$ des cas). La pneumonie varicelleuse touche principalement les sujets fragiles ou immunodéprimés $(90 \%$ des cas) [50]. Différents facteurs de risque sont rapportés chez l'immunocompétent : tabac, sexe masculin et grossesse [51]. Certains travaux rapportent que 10 à $50 \%$ des patients adultes avec une varicelle ont une atteinte pulmonaire [52] ; l'atteinte cutanée précède l'atteinte pulmonaire dans $90 \%$ des cas [51]. La présentation clinique pulmonaire est aspécifique ; les examens radiologiques objectivent un syndrome interstitiel diffus souvent réticulonodulaire parfois à composante alvéolaire [53]. Il s'agit d'une atteinte grave pouvant conduire à un SDRA avec 10 à $50 \%$ de mortalité [54]. Une revue de 2004 sur 120 pneumonies varicelleuses retrouve $31 \%$ de patients nécessitant la mise sous ventilation mécanique et $9 \%$ de mortalité [55]. L'utilisation précoce de l'acyclovir par voie intraveineuse. ( 5 à $10 \mathrm{mg} / \mathrm{kg}$ par 8 heures, sept à dix jours) diminue la mortalité $[51,52]$. En revanche, il n'existe pas de preuve du bénéfice d'une corticothérapie dans cette indication [56]. Concernant les cas de SDRA réfractaires, deux travaux colligent 20 patients avec $60 \%$ de survie sous ECMO [57,58]. 


\section{Hantavirus (famille des Bunyaviridae)}

Hantavirus est un virus rare en France, l'incidence moyenne est de 60 cas par an. La contamination est aérienne à partir d'aérosols ou de poussières contenant les excrétas de rongeurs contaminés. L'incubation est d'une à six semaines. La majorité des cas se présente comme un syndrome viroalgique associé à des troubles visuels et à une thrombopénie évocateurs. Le diagnostic est sérologique. Dans les formes graves peuvent survenir une atteinte myocardique, rénale (fièvre hémorragique avec syndrome rénal) ou pulmonaire (HPS, Hantavirus pulmonary syndrome) plus rare en Europe. La radiographie retrouve des infiltrats interstitiels bilatéraux parfois associés à des condensations alvéolaires, voire un SDRA [53,59]. Exceptionnel en Europe, L'HPS se présente comme une PAC grave le plus souvent avec une mortalité de 20 à $40 \%$ [60]. Une revue de la littérature, recensant 100 cas, retrouve $45 \%$ de recours à la ventilation mécanique et une mortalité de $49 \%$ [55]. Il n'existe, à ce jour, pas de traitement spécifique [61].

\section{Parechovirus (famille des Picornaviridae)}

Il existe 16 types de Parechovirus (HPeV-1 à 16), mais les génotypes 1 à 6 sont les plus fréquents lors des infections des voies respiratoires [62]. La répartition est ubiquitaire, plutôt saisonnière (été-automne) avec un mode de transmission orofécal expliquant sa plus grande prévalence chez les enfants et dans les pays en voie de développement. La principale pathologie due à ces virus est la gastroentérite, mais des complications peuvent survenir : sepsis-like syndrome chez les nourrissons, neurologiques, myocardiques ou encore pulmonaires. Les données de la littérature au sujet des pneumonies à Parechovirus sont pauvres ; une étude rétrospective décrit 28 cas sur 20 ans (dont un seul adulte de 78 ans) sans que le rôle pathogène du Parechovirus soit certain [63].

\section{Cytomégalovirus}

La pneumonie à CMV est quasi exclusivement l'apanage des patients immunodéprimés [64] et n'est jamais rencontrée au cours des pneumonies communautaires.

\section{Herpes simplex virus (HSV)}

La pneumonie à HSV est quasi exclusivement l'apanage des patients immunodéprimés [64], mais quelques cas ont été décrits chez l'immunocompétent présentant des facteurs de risque : tabagisme, brûlé avec atteinte trachéobronchique ou antécédent d'intubation orotrachéale [65]. Elle se présente comme une pneumonie condensante avec des zones de verre dépoli et des lésions trachéobronchiques macroscopiques à l'endoscopie (nécroses, ulcérations) [53].

\section{Epstein-Barr virus (EBV)}

Les PAC graves à EBV de l'adulte immunocompétent sont exceptionnelles. Il existe quelques cas cliniques décrits où une mononucléose infectieuse (à EBV) se complique de PAC virale spécifique confirmée histologiquement [66,67]. Un cas clinique plus récent décrit un SDRA attribué à l'EBV traité par acyclovir et immunoglobulines [68], mais cette entité reste anecdotique.

\section{Human herpes virus (HHV-6 et HHV-7 famille des Herpesviridae)}

Plus de $90 \%$ des adultes sont séropositifs pour HHV-6 et la primo-infection est responsable, lorsqu'elle est symptomatique, de la roséole infantile. La PAC à HHV-6 est décrite [5] mais elle est exceptionnelle chez l'immunocompétent et rare chez l'immunodéprimé. Il existe un cas décrit de SDRA à HHV-6 [69]. Il existe un cas décrit de SDRA à HHV-7 chez l'immunocompétent [70].

\section{Measles virus}

Les infections dues au Measles virus (rougeole entre autres) touchent 30 à 40 millions de personnes par an avec environ 1,5 million de décès par an. Maladie ubiquitaire et épidémique à transmission orofécale, elle touche surtout les enfants et les pays en voie de développement [71]. À l'origine d'un syndrome cutané et digestif, les infections à Measles virus peuvent être responsables d'encéphalites ou de pneumonies virales. Un travail ancien décrit 106 cas $(3,3 \%)$ de pneumonie virale sur 3220 infections à Measles. Aucun cas de décès n'est survenu, mais $30 \%$ de ces patients avaient une coinfection bactérienne (haemophilus, neisseria, streptocoque et moraxella) [72]. Il existe certains facteurs de risque : la grossesse [73] et l'immunodépression [74].

\section{Mimivirus (mimicking microbe virus)}

Initialement assimilé à une bactérie, il a été reclassé en tant que virus en 2003 [75]. Il se développe aux dépens d'une amibe aquatique supposant une contamination environnementale (eau). La physiopathologie et son rôle pathogène ne sont pas clairs ; mais le Mimivirus est responsable de pneumonies virales dans des modèles animaux et chez des patients humains [39,76]. La présentation clinique est aspécifique. Une étude a retrouvé $2 \%$ de prélèvements positifs chez des sujets sains témoins contre $10 \%$ dans des cas de PAC et $20 \%$ de ces patients admis en réanimation, mais les diagnostics étaient sérologiques et rétrospectifs [77]. Un travail récent portant sur 244 cas de PAC n'a retrouvé aucun prélèvement positif à Mimivirus (RT-PCR sur prélèvements des voies aériennes supérieures) [78] ; tous ces résultats 
suggèrent que le Mimivirus n'est pas un agent causal fréquent de PAC.

\section{Pneumonies à micro-organismes atypiques}

Les bactéries atypiques représenteraient jusqu'à $44 \%$ des causes de PAC. Décrites moins graves que les PAC à micro-organismes usuels [79], $25 \%$ de ces pneumonies nécessitent une hospitalisation, $15 \%$ sont en sepsis sévère et $0,7 \%$ sont admis en réanimation. Le Tableau 1 résume les points de la présentation clinique pouvant orienter vers une PAC à bactéries atypiques.

\section{Légionellose}

Legionella pneumophila (Lp) est une bactérie à Gram négatif aérobie non fermentant, flagellée, hydrotellurique et à croissance intracellulaire dont il a été décrit 16 sérotypes. Les sources de contamination sont les systèmes de douches, les climatiseurs et les dispositifs de nébulisation [80]. Les sérogroupes 1 à 6 sont impliqués en pathologie humaine [81]. En Europe, Lp de type 1 est responsable de $90 \%$ de toutes les légionelloses [82,83]. D'après l'EWGLI, près de 12000 nouveaux cas ont été déclarés en Europe entre 2007 et 2008 [82] dont $63 \%$ de PAC, $21 \%$ de pneumonies acquises pendant un voyage (moins fréquentes dans les pays du Sud de l'Europe) et seulement 6,3\% de forme nosocomiale. Lp est impliquée dans moins de $1 \%$ des pneumonies liées aux soins [84]. Les légionelloses surviennent en majorité chez des hommes (70,6\%), et $22 \%$ des patients ont entre 50 et 60 ans avec une incidence croissante avec l'âge. La mortalité attribuable a régressé depuis 30 ans et varie entre 4 et $12 \%$. Elle est de $8,1 \%$ pour les légionelloses hospitalisées [85] et peut atteindre $50 \%$ dans les formes nosocomiales [83]. En réanimation, la mortalité attribuable est de $33 \%$. L'existence de comorbidités, le délai de prise en charge et le caractère nosocomial ont été associés à une surmortalité. Six facteurs prédictifs de mortalité ont été identifiés dans les formes hospitalisées : l'âge, le sexe féminin, l'admission en réanimation, l'insuffisance rénale aiguë, une corticothérapie et une protéine $\mathrm{C}$ réactive supérieure à $500 \mathrm{mg} / 1$ [85]. La gravité est aussi dépendante de la virulence de la souche et de la durée de l'exposition à la source contaminante [80]. Le retard diagnostique et l'inefficacité de l'antibiothérapie initiale sont de mauvais pronostic [86,87] : $47 \%$ des légionelloses en réanimation avaient reçu une première antibiothérapie, le plus souvent amoxicilline plus ou moins clavulanate. L'échec d'un antibiotique antipneumococcique est très évocateur de légionellose. Les symptômes des légionelloses sont aspécifiques, et ce diagnostic doit être évoqué sur la présentation subaiguë et la présence de facteurs de risque de Lp. Les symptômes, souvent extrarespiratoires, évoluent depuis cinq à sept jours au moment du diagnostic. Les facteurs de risque sont le diabète, un tabagisme actif, un voyage récent à l'étranger, un séjour d'au moins une nuit à l'hôtel et la profession de chauffeur de taxi [88]. La légionellose du voyageur survient souvent chez un patient sans comorbidités là où les patients admis en réanimation ont au moins une comorbidité dans $81 \%$ des cas [86]. Certains symptômes ou anomalies clinicobiologiques renforcent la présomption de légionellose : bradycardie relative, diarrhée hydrique douloureuse [89]. Présentes chez $30 \%$ des patients, les céphalées et la confusion sont peu

Tableau 1 Présentations cliniques évocatrices de PAC causées par une bactérie atypique

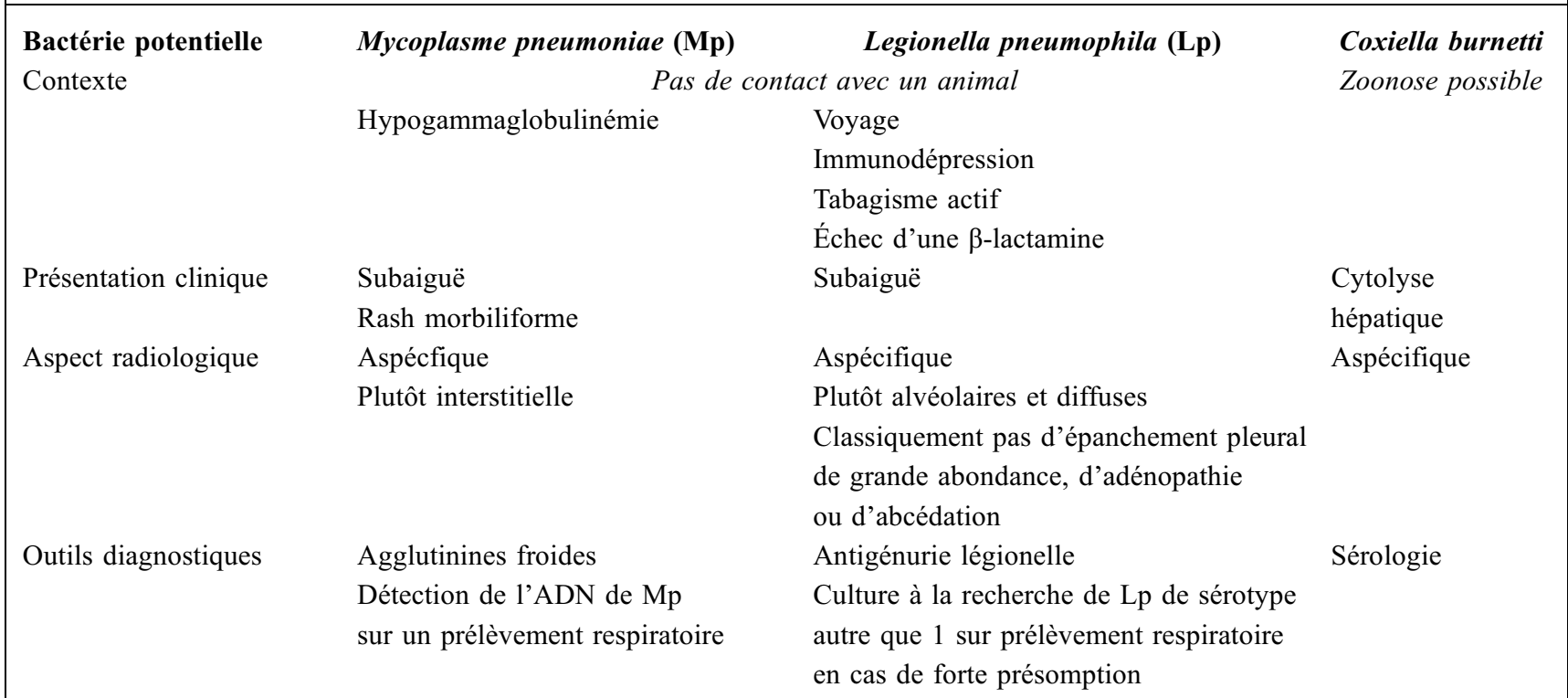


discriminantes. Une rhabdomyolyse, une cytolyse hépatique modérée, une hématurie microscopique et une hypophosphorémie sont très évocatrices de légionellose. Les anomalies radiologiques sont aussi aspécifiques, le tableau classique montre des opacités alvéolo-interstitielles asymétriques « patchy », souvent bilatérales dans les formes graves [90]. Un épanchement pleural abondant, un abcès et une adénopathie hilaire sont exceptionnels. La culture reste la technique de référence. Elle présente de nombreux avantages (spécificité de $100 \%$, détection de tous les sérotypes de Lp) mais nécessite un prélèvement respiratoire, se positive en sept jours et reste peu sensible [83]. Son rendement est meilleur en cas de détresse respiratoire $(90 \%$ de positivité contre $25 \%$ dans les légionelloses tout-venant) où il est recommandé de rechercher une $\mathrm{Lp}$ de sérotype autre que 1 . La sérologie n'est pas utilisée en réanimation, car la séroconversion est tardive (trois à quatre semaines). La détection d'acide désoxyribonucléique (ADN) [91] a été supplantée par l'antigénurie car rapide, accessible, facile d'exécution sur un simple échantillon d'urines. L'antigénurie est positive dès le deuxième jour mais ne détecte que le sous-type Lp1 Pontiac. Une méta-analyse récente a estimé la sensibilité à $74 \%$ et la spécificité à $99 \%$, qui semblent meilleures dans les formes graves [92]. Lp est sensible aux macrolides, aux kétolides, aux fluoroquinolones (FQ), à la rifampicine et aux cyclines. Les macrolides sont historiquement le traitement de référence de la légionellose : l'érythromycine a été utilisée dès 1976. Bien qu'ayant une meilleure concentration minimale inhibitrice (CMI) que l'érythromycine, l'azithromycine intraveineuse n'a pas l'autorisation de mise sur le marché en France. La rifampicine et les FQ ont des CMI inférieures à celles des macrolides [93]. Les données sur le bénéfice des FQ sont contradictoires sur la survenue de complications (admission en réanimation, défaillance d'organe sévère) et la durée de séjour [87,94], aucune étude n'a montré une baisse de mortalité en faveur des FQ. Une méta-analyse a montré l'absence de différence d'efficacité entre les FQ et l'association $\beta$-lactamines-macrolides dans le traitement des PAC, y compris les légionelloses [95]. D'un point de vue pharmacodynamique, la synergie FQ-macrolides a été démontrée. En pratique, on propose un macrolide pour les suspicions de légionelloses, car l'Agence nationale de sécurité du médicament et des produits de santé (Afssaps) limite l'usage des FQ aux seules formes graves documentées. L'Infectious Diseases Society of America (IDSA) [96] et la Société de pathologie infectieuse de langue française (SPILF) recommandent une céphalosporine de troisième génération et un macrolide ou une FQ pour une PAC grave. En cas de légionellose documentée, il est recommandé un arrêt de la $\beta$-lactamine et une association de deux antibiotiques actifs sur Lp dans les formes sévères (Tableau 2) pendant cinq jours puis de maintenir une monothérapie pendant 14 à 21 jours. La rifampicine ne doit pas être utilisée en monothérapie car à fort risque d'acquisition de résistance.

\section{Mycoplasme}

La prévalence de Mycoplasma pneumoniae (Mp) dans les PAC est de $12 \%$ dans le monde [97] et de 15,9\% en Europe [79]. Mp est moins fréquent dans les PAC graves hospitalisées : 8 à $11 \%$ des cas [79,98], et le recours à la ventilation mécanique ne concerne que $11 \%$ des PAC à Mp hospitalisées [99]. La mortalité globale est de 0,7 contre $8,5 \%$ pour les autres étiologies de PAC. Les cas de pneumonies graves à Mp sont donc rares. Si elle survient préférentiellement chez les sujets jeunes, Mp est la deuxième cause de PAC chez le sujet âgé [100]. Il n'existe pas de facteur de risque connu de pneumonie à $\mathrm{Mp}$ en dehors de l'hypogammaglobulinémie. Des cas familiaux graves ont été rapportés [101,102]. La présentation clinique n'est pas spécifique [89]. Il existe dans $25 \%$ des cas un érythème morbilliforme et une atteinte des voies aériennes supérieures (myringite bulleuse, otite, pharyngite) $[89,103]$. Des diarrhées hydriques indolores sont aussi fréquentes. L'anémie hémolytique est rare mais sévère. Elle est allo-immunitaire par réaction croisée d'agglutinines froides, dont la présence est très évocatrice de Mp [89] sans en être spécifiques car décrites dans d'autres infections (rickettsia, adénovirus, virus influenza) ; de forts taux d'agglutinines froides ont été retrouvés dans les formes graves. La présentation radiologique aspécifique est constituée d'infiltrats interstitiels réticulés périhilaires, bilatéraux dans $20 \%$ des cas. Les patients les plus sévères présentent plutôt

Tableau 2 Stratégie du choix antibiotique dans les légionelloses (d'après les recommandations de l'Afsaps)

Gravité de la légionellose/terrain sous-jacent Choix antibiotique

Légionellose non grave (patient ambulatoire

ou hospitalisé en médecine)

Légionellose grave (patient hospitalisé en soins

intensifs ou en réanimation et/ou immunodéprimé)
Monothérapie par macrolide : spriramycine ou érythromycine ou azythromycine ou roxithromycine ou clarithromycine

Monothérapie par fluoroquinolone : lévofloxacine, ofloxacine ou ciprofloxacine

Ou association de 2 antibiotiques parmi : macrolide (disponible par voie parentérale : spiramycine et érythromycine), fluorquinolone (lévofloxacine, ofloxacine ou ciprofloxacine) et rifampicine 
des condensations alvéolaires [103]. La culture de Mp, laborieuse et coûteuse, n'est pas utilisée en routine. La détection d'ADN par PCR est une technique validée sur des modèles animaux, humains et sur différents types de prélèvements [104]. Positive précocement, la PCR se négative rapidement après initiation du traitement [103]. En pratique, elle est réalisée sur un prélèvement respiratoire. La sérologie, très répandue, détecte les $\operatorname{IgG}$ et IgM sur deux prélèvements réalisés à trois semaines d'intervalle; sa sensibilité atteint $90 \%$ et sa spécificité $88 \%$. Une stratégie combinant PCR et sérologie a été utilisée dans le cadre des grandes études épidémiologiques récentes [98,105], car la PCR se négative rapidement après traitement alors que la sérologie qui peut être initialement négative et autorise un diagnostic plus tardif.

Les macrolides sont le traitement de référence des pneumonies à $\mathrm{Mp}$ avec un risque quasi inexistant de résistance. La clarithromycine, l'azithromycine et l'érythromycine ont le même taux de succès clinique (plus $90 \%$ ), mais les deux derniers sont préférés en raison de leur meilleure tolérance, en particulier cardiaque. Concernant les FQ, le risque théorique de résistance est plus important, mais il n'en a jamais été rapporté [103], et leur efficacité est équivalente aux macrolides. Il n'existe aucune recommandation pour le traitement des pneumonies à $\mathrm{Mp}$, en particulier sur le bénéfice d'une bithérapie par macrolide-FQ et d'une corticothérapie systémique. La durée de traitement est de 10 à 15 jours.

\section{Chlamydia pneumoniae}

Les Chlamydia pneumoniae (Cp) semblent impliquées dans $10,6 \%$ des PAC tout-venant et seulement dans 5,6\% des PAC hospitalisées [79]. Il n'est pas décrit de forme sévère de pneumonie à $\mathrm{Cp}$ chez l'adulte en Europe. Aucun élément clinique ne permet de distinguer une pneumonie à $\mathrm{Cp}$ d'une pneumonie à Mp [106]. Il semblerait que l'asthme soit un terrain privilégié, mais cette association a aussi été discutée pour Mp [107]. De nombreuses coinfections ont été décrites sans qu'il soit possible de montrer une association avec des formes sévères [108]. Le diagnostic est sérologique et le traitement repose sur les macrolides.

\section{Autres bactéries atypiques}

Ce sont les zoonoses dont le diagnostic est évoqué essentiellement sur le contexte de contact avec un animal. Les principales zoonoses sont les pneumonies à Chlamydia psittacii, la tularémie et en particulier la fièvre $\mathrm{Q}$ à Coxiella burneti, décrite comme la plus fréquente des pneumonies atypiques en Espagne [79]. Tous les mammifères peuvent être vecteurs de la fièvre $Q$, en particulier les animaux domestiques et les animaux d'élevage [109]. La pneumonie, exceptionnellement grave, est une manifestation aiguë et fait suite à une période d'incubation de trois semaines ; elle est toujours associée à une cytolyse par hépatite granulomateuse. La gravité est due à d'autres complications : anémie hémolytique, thyroïdite, péricardite, myocardite, pancréatite. Le diagnostic est sérologique. Le traitement repose sur les cyclines, en particulier la doxycycline $(200 \mathrm{mg} / \mathrm{j})$ pendant deux semaines. En cas d'allergie, la rifampicine et les FQ peuvent être prescrites.

\section{Conclusion}

L'intérêt que le réanimateur doit avoir au lit du malade visà-vis de certains micro-organismes atypiques et des virus dans les PAC sévères doit rester secondaire, en dehors d'un contexte épidémiologique très précis. En effet, l'antibiothérapie, dans cette situation, reste probabiliste et repose sur une association $\beta$-lactamines et macrolides ou FQ. Seule une documentation microbiologique secondaire précise et fiable permettra de discuter l'arrêt de certains antibiotiques « probabilistes » et la prescription « ciblée » d'anti-infectieux spécifiques des étiologies retrouvées. Si les méthodes diagnostiques pour les bactéries typiques ou atypiques semblent pouvoir donner une certitude de « diagnostic clinique », les méthodes de biologie moléculaire pour les virus posent le problème de l'imputabilité clinique de leur positivité. Si les pneumonies virales « réelles » se révèlent si fréquentes, il est temps de trouver des traitements antiviraux réellement efficaces.

Conflit d'intérêt : les auteurs déclarent ne pas avoir de conflit d'intérêt.

\section{Références}

1. Black RE, Cousens S, Johnson HL, et al (2010) Global, regional, and national causes of child mortality in 2008: a systematic analysis. Lancet 375:1969-87

2. Garcia-Vidal C, Ardanuy C, Tubau F, et al (2010) Pneumococcal pneumonia presenting with septic shock: host- and pathogenrelated factors and outcomes. Thorax 65:77-81

3. el-Ebiary M, Sarmiento X, Torres A, et al (1997) Prognostic factors of severe Legionella pneumonia requiring admission to ICU. Am J Respir Crit Care Med 156:1467-72

4. Lim WS, Baudouin SV, George RC, et al (2009) BTS guidelines for the management of community acquired pneumonia in adults: update 2009. Thorax 64(Suppl 3):iii1-iii55

5. Ruuskanen O, Lahti E, Jennings LC, Murdoch DR (2011) Viral pneumonia. Lancet 377:1264-75

6. Peiris JS, Yuen KY, Osterhaus AD, Stohr K (2003) The severe acute respiratory syndrome. N Engl J Med 349:2431-41

7. Fallone CA (2000) Epidemiology of the antibiotic resistance of Helicobacter pylori in Canada. Can J Gastroenterol 14:879-82

8. Novel Swine-Origin Influenza AVIT, Dawood FS, Jain S, et al (2009) Emergence of a novel swine-origin influenza A (H1N1) virus in humans. N Engl J Med 360:2605-15 
9. Luyt CE, Combes A, Nieszkowska A, et al (2008)Viral infections in the ICU. Curr Opin Crit Care 14:605-8

10. Wong SS, Yuen KY (2008) Antiviral therapy for respiratory tract infections. Respirology 13:950-71

11. Schuetz P, Albrich W, Christ-Crain M, et al (2010) Procalcitonin for guidance of antibiotic therapy. Expert Rev Anti Infect Ther 8:575-87

12. Murdoch DR, Jennings LC, Bhat N, Anderson TP (2010) Emerging advances in rapid diagnostics of respiratory infections. Infect Dis Clin North Am 24:791-807

13. Talbot HK, Falsey AR (2010) The diagnosis of viral respiratory disease in older adults. Clin Infect Dis 50:747-51

14. Tiveljung-Lindell A, Rotzen-Ostlund M, Gupta S, et al (2009) Development and implementation of a molecular diagnostic platform for daily rapid detection of 15 respiratory viruses. J Med Virol 81:167-75

15. Lieberman D, Shimoni A, Keren-Naus A, et al (2009) Identification of respiratory viruses in adults: nasopharyngeal versus oropharyngeal sampling. J Clin Microbiol 47:3439-43

16. Garbino J, Soccal PM, Aubert JD, et al (2009) Respiratory viruses in bronchoalveolar lavage: a hospital-based cohort study in adults. Thorax 64:399-404

17. Choi SH, Hong SB, Ko GB, et al (2012) Viral infection in patients with severe pneumonia requiring intensive care unit admission. Am J Respir Crit Care Med 186:325-32

18. Jennings LC, Anderson TP, Beynon KA, et al (2008) Incidence and characteristics of viral community-acquired pneumonia in adults. Thorax 63:42-8

19. Johnstone J, Majumdar SR, Fox JD, Marrie TJ (2008) Viral infection in adults hospitalized with community-acquired pneumonia: prevalence, pathogens, and presentation. Chest 134:1141-8

20. Lieberman D, Shimoni A, Shemer-Avni Y, et al (2010) Respiratory viruses in adults with community-acquired pneumonia. Chest 138:811-6

21. Hopkins P, McNeil K, Kermeen F, et al (2008) Human metapneumovirus in lung transplant recipients and comparison to respiratory syncytial virus. Am J Respir Crit Care Med 178:876-81

22. Kumar A (2011) Early versus late oseltamivir treatment in severely ill patients with 2009 pandemic influenza A (H1N1): speed is life. J Antimicrob Chemother 66:959-63

23. Writing Committee of the WHOCoCAoPI, Bautista E, Chotpitayasunondh T, et al (2010) Clinical aspects of pandemic 2009 influenza A (H1N1) virus infection. N Engl J Med 362:1708-19

24. Beigel JH, Farrar J, Han AM, et al (2005) Avian influenza A (H5N1) infection in humans. N Engl J Med 353:1374-85

25. Australia and New Zealand Extracorporeal Membrane Oxygenation (ANZ ECMO) Influenza Investigators, Davies A, Jones D, Bailey M, Beca J, et al (2009) Extracorporeal Membrane Oxygenation for 2009 Influenza A(H1N1) Acute Respiratory Distress Syndrome. JAMA 302:1888-95

26. Brun-Buisson C, Richard JC, Mercat A, et al (2011) Early corticosteroids in severe influenza A/H1N1 pneumonia and acute respiratory distress syndrome. Am J Respir Crit Care Med 183:1200-6

27. Dowell SF, Anderson LJ, Gary HE Jr, et al (1996) Respiratory syncytial virus is an important cause of community-acquired lower respiratory infection among hospitalized adults. J Infect Dis $174: 456-62$

28. Walsh EE, Peterson DR, Falsey AR (2004) Risk factors for severe respiratory syncytial virus infection in elderly persons. J Infect Dis 189:233-8

29. Clark TW, Fleet DH, Wiselka MJ (2011) Severe communityacquired adenovirus pneumonia in an immunocompetent 44-year-old woman: a case report and review of the literature. J Med Case Rep 5:259
30. Kandel R, Srinivasan A, D'Agata EM, et al (2010) Outbreak of adenovirus type 4 infection in a long-term care facility for the elderly. Infect Control Hosp Epidemiol 31:755-7

31. Esposito DH, Gardner TJ, Schneider E, et al (2010) Outbreak of pneumonia associated with emergent human adenovirus serotype 14--Southeast Alaska, 2008. J Infect Dis 202:214-22

32. Zhong NS, Zheng BJ, Li YM, et al (2003) Epidemiology and cause of severe acute respiratory syndrome (SARS) in Guangdong, People's Republic of China, in February, 2003. Lancet 362:1353-8

33. Marx A, Gary HE, Jr., Marston BJ, et al (1999) Parainfluenza virus infection among adults hospitalized for lower respiratory tract infection. Clin Infect Dis 29:134-40

34. Johnston SL, Pattemore PK, Sanderson G, et al (1995) Community study of role of viral infections in exacerbations of asthma in 9-11 year old children. BMJ 310:1225-9

35. Don M, Fasoli L, Paldanius M, et al (2005) Aetiology of community-acquired pneumonia: serological results of a paediatric survey. Scand J Infect Dis 37:806-12

36. Hohenthal $U$, Vainionpaa R, Nikoskelainen $J$, Kotilainen $P$ (2008) The role of rhinoviruses and enteroviruses in community acquired pneumonia in adults. Thorax 63:658-9

37. Centers for Disease C, Prevention (2011) Clusters of acute respiratory illness associated with human enterovirus 68--Asia, Europe, and United States, 2008-2010. MMWR Morb Mortal Wkly Rep 60:1301-4

38. Guo L, Gonzalez R, Xie Z, et al (2011) Bocavirus in children with respiratory tract infections. Emerg Infect Dis 17:1775-7

39. Kahn JS, Kesebir D, Cotmore SF, et al (2008) Seroepidemiology of human bocavirus defined using recombinant virus-like particles. J Infect Dis 198:41-50

40. Longtin J, Bastien M, Gilca R, et al (2008) Human bocavirus infections in hospitalized children and adults. Emerg Infect Dis 14:217-21

41. Kesebir D, Vazquez M, Weibel C, et al (2006) Human bocavirus infection in young children in the United States: molecular epidemiological profile and clinical characteristics of a newly emerging respiratory virus. J Infect Dis 194:1276-82

42. Fry AM, Lu X, Chittaganpitch M, et al (2007) Human bocavirus: a novel parvovirus epidemiologically associated with pneumonia requiring hospitalization in Thailand. J Infect Dis 195:1038-45

43. Jartti T, Hedman K, Jartti L, et al (2012) Human bocavirus-the first 5 years. Rev Med Virol 22:46-64

44. Liu L, Bastien N, Sidaway F, et al (2007) Seroprevalence of human metapneumovirus (hMPV) in the Canadian province of Saskatchewan analyzed by a recombinant nucleocapsid proteinbased enzyme-linked immunosorbent assay. J Med Virol 79:308-13

45. Boivin G, Mackay I, Sloots TP, et al (2004) Global genetic diversity of human metapneumovirus fusion gene. Emerg Infect Dis 10:1154-7

46. Hamelin ME, Cote S, Laforge J, et al (2005) Human metapneumovirus infection in adults with community-acquired pneumonia and exacerbation of chronic obstructive pulmonary disease. Clin Infect Dis 41:498-502

47. Walsh EE, Peterson DR, Falsey AR (2008) Human metapneumovirus infections in adults: another piece of the puzzle. Arch Intern Med 168:2489-96

48. Johnstone J, Majumdar SR, Fox JD, Marrie TJ (2008) Human metapneumovirus pneumonia in adults: results of a prospective study. Clin Infect Dis 46:571-4

49. Deffrasnes C, Hamelin ME, Boivin G (2007) Human metapneumovirus. Semin Respir Crit Care Med 28:213-21

50. Feldman S, Stokes DC (1987) Varicella zoster and herpes simplex virus pneumonias. Semin Respir Infect 2:84-94 
51. Chiner E, Ballester I, Betlloch I, et al (2010) Varicella-zoster virus pneumonia in an adult population: has mortality decreased? Scand J Infect Dis 42:215-21

52. Mohsen AH, McKendrick M (2003) Varicella pneumonia in adults. Eur Respir J 21:886-91

53. Kim EA, Lee KS, Primack SL, et al (2002) Viral pneumonias in adults: radiologic and pathologic findings. Radiographics 22 $\left(\right.$ Spec $\left.\mathrm{n}^{\circ}\right):$ S137-S49

54. Nilsson A, Ortqvist A (1996) Severe varicella pneumonia in adults in Stockholm County 1980-1989. Scand J Infect Dis 28:121-3

55. Cheng VC, Tang BS, Wu AK, et al (2004) Medical treatment of viral pneumonia including SARS in immunocompetent adult. J Infect 49:262-73

56. Ahmed R, Ahmed QA, Adhami NA, Memish ZA (2002) Varicella pneumonia: another 'steroid responsive' pneumonia? J Chemother 14:220-2

57. Roberts N, Peek GJ, Jones N, et al (2002) Deaths from Chickenpox. Extracorporeal membrane oxygenation has important role. BMJ 324:610-1

58. Lee WA, Kolla S, Schreiner RJ, Jr., et al (1997) Prolonged extracorporeal life support (ECLS) for varicella pneumonia. Crit Care Med 25:977-82

59. Colby TV, Zaki SR, Feddersen RM, Nolte KB (2000) Hantavirus pulmonary syndrome is distinguishable from acute interstitial pneumonia. Arch Pathol Lab Med 124:1463-6

60. Simpson SQ, Spikes L, Patel S, Faruqi I (2010) Hantavirus pulmonary syndrome. Infect Dis Clin North Am 24:159-73

61. Miedzinski L (2005) Community-acquired pneumonia: new facets of an old disease--Hantavirus pulmonary syndrome. Respir Care Clin N Am 11:45-58

62. Ren L, Xiang Z, Guo L, Wang J (2012) Viral infections of the lower respiratory tract. Curr Infect Dis Rep 14:284-91

63. Abed Y, Boivin G (2006) Human parechovirus types 1, 2 and 3 infections in Canada. Emerg Infect Dis 12:969-75

64. Cesario TC (2012) Viruses associated with pneumonia in adults. Clin Infect Dis 55:107-13

65. Graham BS, Snell JD, Jr (1983) Herpes simplex virus infection of the adult lower respiratory tract. Medicine (Baltimore) 62:384-93

66. Myers JL, Peiper SC, Katzenstein AL (1989) Pulmonary involvement in infectious mononucleosis: histopathologic features and detection of Epstein-Barr virus-related DNA sequences. Mod Pathol 2:444-8

67. Sriskandan S, Labrecque LG, Schofield J (1996) Diffuse pneumonia associated with infectious mononucleosis: detection of Epstein-Barr virus in lung tissue by in situ hybridization. Clin Infect Dis 22:578-9

68. Riachy M, Baaklini C, Ibrahim I, et al (2007) Acute respiratory distress syndrome complicating Ebstein-Barr virus pneumonia. Rev Mal Respir 24:639-43

69. Merk J, Schmid FX, Fleck M, et al (2005) Fatal pulmonary failure attributable to viral pneumonia with human herpes virus 6 (HHV6) in a young immunocompetent woman. J Intensive Care Med 20:302-6

70. Costa C, Bergallo M, Delsedime L, et al (2009) Acute respiratory distress syndrome associated with HHV-7 infection in an immunocompetent patient: a case report. New Microbiol 32:315-6

71. Garly ML, Bale C, Martins CL, et al (2006) Prophylactic antibiotics to prevent pneumonia and other complications after measles: community based randomised double blind placebo controlled trial in Guinea-Bissau. BMJ 333:1245

72. Gremillion DH, Crawford GE (1981) Measles pneumonia in young adults. An analysis of 106 cases. Am J Med 71:539-42

73. Eberhart-Phillips JE, Frederick PD, Baron RC, Mascola L (1993) Measles in pregnancy: a descriptive study of 58 cases. Obstet Gynecol 82:797-801
74. Kaplan LJ, Daum RS, Smaron M, McCarthy CA (1992) Severe measles in immunocompromised patients. JAMA 267:1237-41

75. La Scola B, Audic S, Robert C, et al (2003) A giant virus in amoebae. Science 299:2033

76. Raoult D, La Scola B, Birtles R (2007) The discovery and characterization of Mimivirus, the largest known virus and putative pneumonia agent. Clin Infect Dis 45:95-102

77. La Scola B, Marrie TJ, Auffray JP, Raoult D (2005) Mimivirus in pneumonia patients. Emerg Infect Dis 11:449-52

78. Dare RK, Chittaganpitch M, Erdman DD (2008) Screening pneumonia patients for mimivirus. Emerg Infect Dis 14:465-7

79. Capelastegui A, Espana PP, Bilbao A, et al (2012) Etiology of community-acquired pneumonia in a population-based study: Link between etiology and patients characteristics, processof-care, clinical evolution and outcomes. BMC Infect Dis 12:134

80. Che D, Campese C, Jarraud S (2011) Legionella and legionnaires' disease: what do we know? Pathol Biol (Paris) 59:134-6

81. Cunha BA (2010) Legionnaires' disease: clinical differentiation from typical and other atypical pneumonias. Infect Dis Clin North Am 24:73-105

82. Joseph CA, Ricketts KD, European Working Group for Legionella I (2010) Legionnaires disease in Europe 2007-2008. Euro Surveill 15:19493

83. Diederen BM (2008) Legionella spp. and Legionnaires' disease. J Infect 56:1-12

84. Miyashita N, Kawai Y, Akaike H, et al (2012) Clinical features and the role of atypical pathogens in nursing and healthcareassociated pneumonia (NHCAP): differences between a teaching university hospital and a community hospital. Intern Med 51:585-94.

85. Chidiac C, Che D, Pires-Cronenberger S, et al (2012) Factors associated with hospital mortality in community-acquired legionellosis in France. Eur Respir J 39:963-70

86. Gacouin A, Le Tulzo Y, Lavoue S, et al (2002) Severe pneumonia due to Legionella pneumophila: prognostic factors, impact of delayed appropriate antimicrobial therapy. Intensive Care Med 28:686-91

87. Mykietiuk A, Carratala J, Fernandez-Sabe N, et al (2005) Clinical outcomes for hospitalized patients with Legionella pneumonia in the antigenuria era: the influence of levofloxacin therapy. Clin Infect Dis 40:794-9

88. Den Boer JW, Nijhof J, Friesema I (2006) Risk factors for sporadic community-acquired Legionnaires' disease. A 3-year national case-control study. Public Health 120:566-71

89. Cunha BA (2006) The atypical pneumonias: clinical diagnosis and importance. Clin Microbiol Infect 12(Suppl 3):12-24

90. Boersma WG, Daniels JM, Lowenberg A, et al (2006) Reliability of radiographic findings and the relation to etiologic agents in community-acquired pneumonia. Respir Med 100:926-32

91. Murdoch DR (2004) Molecular genetic methods in the diagnosis of lower respiratory tract infections. APMIS 112:713-27

92. Shimada T, Noguchi Y, Jackson JL, et al (2009) Systematic review and metaanalysis: urinary antigen tests for Legionellosis. Chest 136:1576-85

93. Gomez-Lus R, Adrian F, del Campo R, et al (2001) Comparative in vitro bacteriostatic and bactericidal activity of trovafloxacin, levofloxacin and moxifloxacin against clinical and environmental isolates of Legionella spp. Int J Antimicrob Agents 18:49-54

94. Blazquez Garrido RM, Espinosa Parra FJ, Alemany Frances L, et al (2005) Antimicrobial chemotherapy for Legionnaires disease: levofloxacin versus macrolides. Clin Infect Dis 40:800-6

95. Vardakas KZ, Siempos, II, Grammatikos A, et al (2008) Respiratory fluoroquinolones for the treatment of community-acquired pneumonia: a meta-analysis of randomized controlled trials. CMAJ 179:1269-77 
96. Mandell LA, Wunderink RG, Anzueto A, et al (2007) Infectious Diseases Society of America/American Thoracic Society consensus guidelines on the management of communityacquired pneumonia in adults. Clin Infect Dis 44(Suppl 2): S27-S72

97. Arnold FW, Summersgill JT, Lajoie AS, et al (2007) A worldwide perspective of atypical pathogens in community-acquired pneumonia. Am J Respir Crit Care Med 175:1086-93

98. von Baum H, Welte T, Marre R, et al (2009) Mycoplasma pneumoniae pneumonia revisited within the German Competence Network for Community-acquired pneumonia (CAPNETZ). BMC Infect Dis 9:62

99. Marrie TJ, Peeling RW, Reid T, et al (2003) Chlamydia species as a cause of community-acquired pneumonia in Canada. Eur Respir J 21:779-84

100. Takiguchi Y, Shikama N, Aotsuka N, et al (2001) Fulminant Mycoplasma pneumoniae pneumonia. Intern Med 40:345-8

101. Kannan TR, Hardy RD, Coalson JJ, et al (2012) Fatal outcomes in family transmission of Mycoplasma pneumoniae. Clin Infect Dis $54: 225-31$

102. Chaudhry R, Tabassum I, Kapoor L, et al (2010) A fulminant case of acute respiratory distress syndrome associated with
Mycoplasma pneumoniae infection. Indian J Pathol Microbiol 53:555-7

103. Waites KB, Talkington DF (2004) Mycoplasma pneumoniae and its role as a human pathogen. Clin Microbiol Rev 17:697-728, [table of contents]

104. Daxboeck F, Khanakah G, Bauer C, et al (2005) Detection of Mycoplasma pneumoniae in serum specimens from patients with Mycoplasma pneumonia by PCR. Int J Med Microbiol 295:279-85

105. Johansson N, Kalin M, Tiveljung-Lindell A, et al (2010) Etiology of community-acquired pneumonia: increased microbiological yield with new diagnostic methods. Clin Infect Dis 50:202-9

106. Miyashita N, Fukano H, Okimoto N, et al (2002) Clinical presentation of community-acquired Chlamydia pneumoniae pneumonia in adults. Chest 121:1776-81

107. Blasi F, Tarsia P, Aliberti S (2009) Chlamydophila pneumoniae. Clin Microbiol Infect 15:29-35

108. Bianco A, Parrella R, Esposito V, et al (2011) Severe A(H1N1)associated Pneumonia sequential to Clamidophila pneumoniae infection in healthy subject. In Vivo 25:825-8

109. Walker DH, Fishbein DB (1991) Epidemiology of rickettsial diseases. Eur J Epidemiol 7:237-45 